\title{
Effective Field Theory Dimensional Regularization
}

\author{
Dirk Lehmann, Gary Prézeau ${ }^{\dagger}$ \\ Department of Physics, Hampton University, \\ Hampton, VA 23668, USA
}

November 4, 2018

\begin{abstract}
A Lorentz-covariant regularization scheme for effective field theories with an arbitrary number of propagating heavy and light particles is given. This regularization scheme leaves the low-energy analytic structure of Greens functions intact and preserves all the symmetries of the underlying Lagrangian. The power divergences of regularized loop integrals are controlled by the low-energy kinematic variables. Simple diagrammatic rules are derived for the regularization of arbitrary one-loop graphs and the generalization to higher loops is discussed.
\end{abstract}

PACS:11.10.-z, 11.15.Bt, 12.39.Hg, 12.39.Fe

\section{Introduction}

Effective Field Theory (EFT) is a consistent framework for calculating Greens functions below a certain scale $\Lambda_{\mathrm{EFT}}$ when the fundamental theory is unsolvable. It is based on the principle that a physical amplitude can be calculated from the most general Lagrangian formulated with the relevant degrees of freedom and consistent with the symmetries of the fundamental theory. EFT then provides a systematic method of organizing an expansion in $p / \Lambda_{\mathrm{EFT}}$ where $p$ is a generic kinematic variable much smaller than $\Lambda_{\mathrm{EFT}}$. This expansion requires a regularization scheme which preserves the power counting in the small kinematic variables.

EFT has been applied successfully to the calculation of Greens functions that involve strongly interacting particles at low-energies [1]. One example is chiral perturbation theory $(\chi \mathrm{PT})$ [2] where only quasi-Goldstone bosons of generic

*dlehmann@jlab.org

†prezeau@jlab.org

¥Mail address: Thomas Jefferson National Accelerator Facility, 12000 Jefferson Avenue, M.S. 12H2, Newport News, VA 23606, USA 
mass $m$ are present and all the kinematic variables are small relative to $\Lambda_{\chi} \approx$ $1 \mathrm{GeV}$. Dimensional regularization can be thought of as a natural regularization scheme for $\chi \mathrm{PT}$, because dimensionally regularized loop integrals can only yield terms that involve powers of the small kinematic variables such as $m / \Lambda_{\chi}$. . Hence, dimensional regularization is a preferred regularization scheme because it allows the assignment of a chiral power to any Feynman graph through a naïve dimensional analysis while preserving the symmetries of the problem.

In the presence of heavy particles with generic masses denoted by $M \gtrsim \Lambda_{\mathrm{EFT}}$, naïve dimensional analysis is no longer valid because the loop integrals also yield terms of the form $M / \Lambda_{\mathrm{EFT}}$ which spoil power counting [3].

For a single heavy particle at low energies, the dependence on $M$ can be removed from the propagators by expanding the EFT Lagrangian in inverse powers of the heavy mass as is done in heavy quark effective field theory and heavy baryon chiral perturbation theory [⿶] The $1 / M$ expansion yields loop integrals that satisfy low-energy power counting. However, the $1 / M$ expansion does not always reproduce the correct low-energy analytic structure. An example of this is the scalar form-factor of the nucleon where the $1 / M$ expansion fails to reproduce the anomalous threshold [5]. Therefore, the $1 / M$ expansion near the anomalous threshold will fail to converge and an infinite number of terms must be re-summed. This re-summation restores manifest Lorentz-invariance. The retention of manifest Lorentz-invariance from the beginning avoids these problems.

A manifestly Lorentz-invariant regularization scheme which preserves the low-energy analytic structure without spoiling power counting is desirable. In the presence of a single heavy particle, such a regularization scheme called infrared regularization was devised for one-loop Feynman graphs by Becher and Leutwyler [6] building on work by Ellis and Tang [7]. The underlying idea of infrared regularization is the separation of power-counting violating terms from the dimensionally regularized loop integrals, and their absorption into the low-energy constants (LECs) of the EFT. The power-counting violating terms are proportional to fractional powers of $M$ which involve non-integer values of the dimension $d$. For example, for the nucleon self-energy graph of Fig. 1, the infrared regularization separates out terms of the form $M^{d-4} /(d-4)$. This separation is achieved by extending the integration domain of the Feynman parameters appearing in Greens functions. Inspired by this work, a regularization of one-loop graphs with two propagating heavy particles was recently developed in Ref. [8] and referred to there as EFT dimensional regularization (EFTDR).

In this paper, a consistent, Lorentz-invariant, natural regularization scheme is presented which generalizes EFTDR to an arbitrary number of heavy and light particles and to higher loops. This regularization systematically separates out all terms involving fractional powers of $M$ implementing a low-energy power counting scheme. To one-loop order, it is proven that the low-energy analytic structure of the Greens functions is left intact by this procedure such that the regularization is valid throughout the low-energy region. It is demonstrated in

\footnotetext{
${ }^{1}$ In contrast to cutoff regularization which introduces powers of the cutoff.
} 


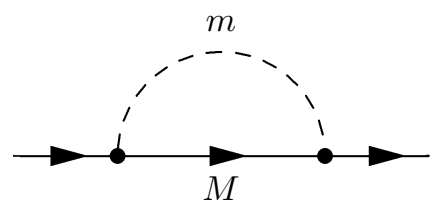

Figure 1: Contribution to the nucleon self energy.

the appendix that this general formalism reproduces the one and two heavy particle sectors as first derived in Refs. 6, 8.

In Sect. 2, the analytic properties of Feynman graphs are reviewed employing the Landau equations. It is shown that the singularities of Feynman integrals can be classified according to subgraphs of the original graph. In Sect. 3, the regularization scheme is introduced. It is shown that the fractional powers of $M$ can be associated with subgraphs of the original graph and can be systematically separated out. To one-loop, simple diagrammatic rules are given to achieve this separation. The application of the formalism to multi-loop graphs is discussed. The results are summarized in Sect. 因.

\section{Analytic structure of Feynman graphs}

Consider a Feynman graph $G$ with $I$ scalar internal lines and $L$ loops in $d$ space-time dimensions,

$$
I_{G}=\mu^{L(4-d)} \int \prod_{\ell=1}^{L} \frac{\mathrm{d}^{d} k_{\ell}}{(2 \pi)^{d}} \prod_{i=1}^{I} \frac{\mathrm{i}}{q_{i}^{2}-m_{i}^{2}+\mathrm{i} \epsilon},
$$

where $\mu$ is the renormalization scale of dimensional regularization. The momentum $q_{i}^{\mu}$ of each internal line $i$ is a linear combination of the loop momenta $k_{\ell}^{\mu}$ and the external momenta $P_{v}^{\mu}$. The Feynman graph thus depends on the particle masses $m_{i}$ and a minimal set of scalar combinations $P_{v} \cdot P_{v^{\prime}}$ of the external momenta. In a general EFT, fermion propagators and derivative couplings give rise to a momentum-dependent numerator in Eq. (1). Since the analytic properties of a Feynman graph only depend on the structure of the denominator as will be shown below, momentum dependent numerators can be neglected for the purpose of this paper. This simplification is not a limitation of the formalism as Feynman graphs involving momentum dependent numerators can always be decomposed into a combination of integrals of the form given by Eq. (1). 
The propagators of Eq. (11) can be combined with Feynman integrals to yield

$$
I_{G}=\mathrm{i}^{I} \Gamma(I) \mu^{L(4-d)} \int_{-\infty}^{\infty} \prod_{k=1}^{I}\left[\mathrm{~d} \alpha_{k} \theta\left(\alpha_{k}\right)\right] \int \prod_{\ell=1}^{L} \frac{\mathrm{d}^{d} k_{\ell}}{(2 \pi)^{d}} \frac{\delta\left(1-\sum_{j=1}^{I} \alpha_{j}\right)}{\left[\sum_{i=1}^{I} \alpha_{i}\left(q_{i}^{2}-m_{i}^{2}\right)+\mathrm{i} \epsilon\right]^{I}},
$$

where the $\theta$-functions have been introduced for later convenience. In general, singularities of $I_{G}$ will arise when the contour of integration gets pinched between two or more poles of the integrand (pinch singularities) or between poles of the integrand and endpoints of the integration contour (endpoint singularities). In either case, the integration contour cannot be distorted away from the pole of the integrand resulting in a singularity of the integral itself.

A useful tool for investigating analytic properties of Feynman graphs is given by the set of Landau equations. They constitute a necessary but not sufficient condition for the occurrence of singularities in $I_{G}$ and their solution determines the locations of the singularities in the space of masses and external momenta as well as their locations in the space of integration variables $\alpha_{i}$. In the representation (2) of the Feynman graph, the Landau equations for $I_{G}$ are given by

$$
\begin{array}{ll}
\text { for each } i: & \text { either } \quad \alpha_{i}=0 \quad \text { or } \quad q_{i}^{2}=m_{i}^{2}, \\
\text { for each } \ell, \mu: & \sum_{i=1}^{I} \alpha_{i} \frac{\partial q_{i}^{2}}{\partial k_{\ell}^{\mu}}=0,
\end{array}
$$

where the $\alpha_{i}$ 's must be normalized to satisfy the $\delta$-function constraint,

$$
\sum_{i=1}^{I} \alpha_{i}=1 .
$$

An immediate consequence of Eq. (3) is the vanishing of the denominator in Eq. (2), $\sum_{i=1}^{I} \alpha_{i}\left(q_{i}^{2}-m_{i}^{2}\right)=0$. Eq. (3) means that an internal particle $i$ of the Feynman graph $G$ is either on-shell or the Feynman parameter $\alpha_{i}$ of line $i$ is zero. If $\alpha_{i}=0$, line $i$ does not contribute to the remaining Landau equations (14). In this case, the set of Landau equations for $G$ is identical to the Landau equations for the subgraph $g$ of $G$ that is obtained by contracting line $i$ to a point. ${ }^{3}$

A solution to the Landau equations where all the $\alpha_{j}$ 's are non-zero, i.e., all internal lines are on-shell, is called the leading singularity of $G$; singularities with $n$ internal lines off-shell are called $(s u b)^{n}$-leading singularities. It follows that

\footnotetext{
${ }^{2}$ See Refs. [9, 10] for a review.

${ }^{3}$ It is important to emphasize that the subgraphs thus obtained are not Feynman graphs and do not represent physical amplitudes. Henceforth, these subgraphs will be represented by lower-case letters.
} 
every (sub) ${ }^{n}$-leading singularity of a Feynman graph $G$ can be represented by the leading singularity of a subgraph $g$ where the corresponding $n$ internal lines of the original graph have been contracted. The complete set of singularities of a Feynman graph $G$ is then given by the leading singularities of each of its subgraphs.

For one-loop graphs, a more practical formulation of the Landau equations can be derived. Routing all internal momenta $q_{i}^{\mu}$ to flow around the loop, Eq. (4) takes on the form

$$
\text { for each } i \text { with } \alpha_{i} \neq 0: \quad \sum_{j=1}^{I} \alpha_{j} q_{j}^{\mu}=0
$$

where only uncontracted lines $j$ contribute. Multiplying this equation by $q_{i \mu}$ for each uncontracted line $i$ yields a set of linear equations

$$
\sum_{j=1}^{I}\left(q_{i} \cdot q_{j}\right) \alpha_{j}=0
$$

Using the on-shell condition (3), the matrix $\left(q_{i} \cdot q_{j}\right)$ can be re-written as

$$
\Omega_{i j}=\left(q_{i} \cdot q_{j}\right)=\frac{m_{i}^{2}+m_{j}^{2}-\left(q_{i}-q_{j}\right)^{2}}{2},
$$

which is now independent of the loop momentum $k^{\mu}$. The location of the singularity in the space of masses and external momenta is determined from the condition

$$
\operatorname{det} \Omega_{i j}=0 \text {, }
$$

while the corresponding location in Feynman parameter space is obtained by solving the zero-eigenvalue problem (7) and normalizing the $\alpha_{i}$ 's according to Eq. (5). A singularity does not arise on the physical sheet when the solution in parameter space lies outside the domain of integration of the Feynman graph.

\subsection{An example: the triangle graph}

As an illustration consider the triangle graph shown in Fig. 2(a) where the solid lines represent heavy particles of mass $M$ and the dashed line represents a light particle of mass $m$. The corresponding subgraphs are shown in Figs. 2(b)-(g).

For the triangle graph, the $\delta$-function appearing in Eq. (2) constrains the domain of integration to a plane in the $\left(\alpha_{1}, \alpha_{2}, \alpha_{3}\right)$-space. Fig. 3 shows the integration domain and the locations of the singularities in this plane. The integration domain (light shaded area) is bounded by the lines $\alpha_{i}=0$. A (sub) ${ }^{1}$ leading singularity corresponding to the subgraph where line $i$ is contracted lies on the line $\alpha_{i}=0$. A (sub) ${ }^{2}$-leading singularity represented by the subgraphs where lines $i$ and $j$ are contracted lies at the intersection of the lines $\alpha_{i}=0$ and $\alpha_{j}=0$ which is a corner of the integration domain. Finally, the Feynman 


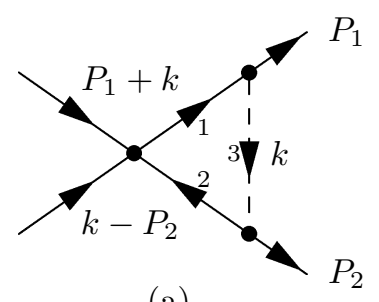

(a)

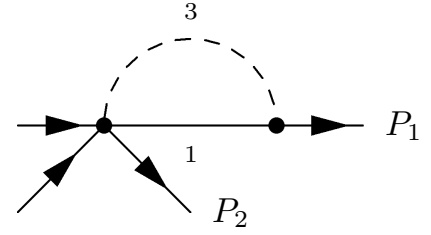

(c)

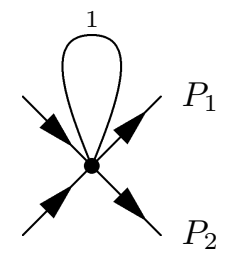

(e)

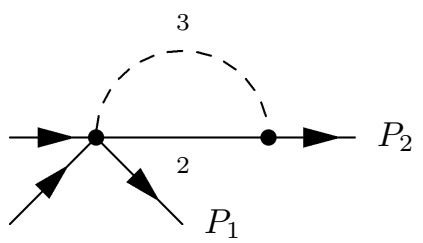

(b)

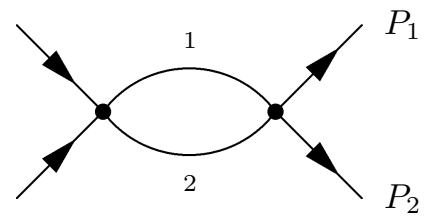

(d)

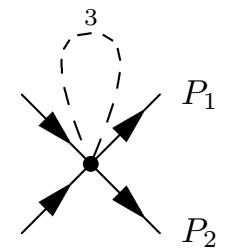

(g)

Figure 2: Triangle graph (a) and all its subgraphs (b)-(g).

parameters that correspond to the leading singularity form a surface given by the dark shaded area in Fig. 3 which penetrates the integration boundaries at the location of the $(\mathrm{sub})^{1}$-leading singularities. In Feynman parameter space, the leading singularity is a pinch singularity while all the sub-leading singularities occurring on the physical sheet lie on the integration boundary and are therefore endpoint singularities.

It is instructive to solve the Landau equations for each subgraph:

- $(S u b)^{2}$-leading singularities. For the heavy-particle tadpole subgraph Fig. 2(e) with lines 2 and 3 contracted, Eqs. (7) and (8) yield immediately

$$
\begin{aligned}
& \alpha_{1} \neq 0, \quad \alpha_{2}=\alpha_{3}=0, \\
& \alpha_{1} M^{2}=0 \quad \Rightarrow M^{2}=0 .
\end{aligned}
$$

Similarly, the light-particle tadpole Fig. 2( $(\mathrm{g})$ yields a singularity at $m^{2}=0$. In Fig. 3, the heavy-particle tadpole singularities are denoted by $\otimes$, the light-particle tadpole by

- $(\text { Sub })^{1}$-leading singularities. For the self-energy subgraph in Fig. 2(b) 


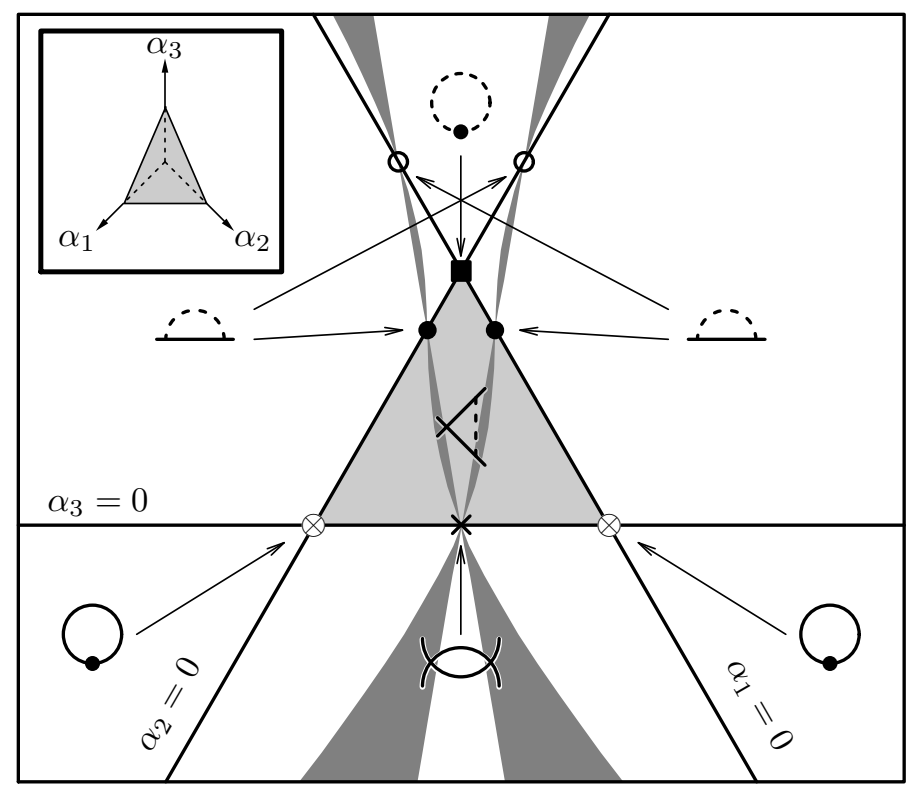

Figure 3: Integration domain and location of singularities of the triangle graph.

condition (9) reads

$$
\begin{aligned}
& \alpha_{1}=0, \quad \alpha_{2} \neq 0, \quad \alpha_{3} \neq 0 \\
& \left|\begin{array}{cc}
M^{2} & \frac{M^{2}+m^{2}-P_{2}^{2}}{2} \\
\frac{M^{2}+m^{2}-P_{2}^{2}}{2} & m^{2}
\end{array}\right|=\left[P_{2}^{2}-(M+m)^{2}\right]\left[P_{2}^{2}-(M-m)^{2}\right]=0 .
\end{aligned}
$$

The corresponding zero-eigenvectors are easily determined. The singularities are located at

$$
\begin{aligned}
& P_{2}^{2}=(M+m)^{2} \quad \text { for } \quad \alpha_{2}=\frac{m}{M+m}, \alpha_{3}=\frac{M}{M+m}, \\
& P_{2}^{2}=(M-m)^{2} \quad \text { for } \quad \alpha_{2}=-\frac{m}{M-m}, \alpha_{3}=\frac{M}{M-m} .
\end{aligned}
$$

Solution (12) (denoted by • in Fig. 3) is recognized as the physical heavylight-threshold, while the pseudo-threshold (13) (denoted by o) does not appear as a singularity on the physical sheet since it lies outside $\left(\alpha_{2}<\right.$ 0 ) the domain of integration of the Feynman graph (2). Similarly, the thresholds for the bubble subgraph in Fig. 2(d) are

$$
\begin{array}{ll}
P^{2}=4 M^{2} & \text { for } \quad \alpha_{1}=\alpha_{2}=\frac{1}{2}, \quad \alpha_{3}=0, \quad(\times \text { in Fig. } 3) ; \\
P^{2}=0 & \text { not on the physical sheet }{ }^{4} .
\end{array}
$$


- Leading Singularity. The leading singularity of the triangle graph, corresponding to $\alpha_{i} \neq 0(i=1,2,3)$, is a manifold rather than a point in the space of independent external momenta $P_{1}^{2}, P_{2}^{2}$, and $P^{2}=\left(P_{1}+P_{2}\right)^{2}$ and satisfies

$$
\left|\begin{array}{ccc}
M^{2} & \frac{2 M^{2}-P^{2}}{2} & \frac{M^{2}+m^{2}-P_{1}^{2}}{M^{2}} \\
\frac{2 M^{2}-P^{2}}{2} & M^{2} & \frac{M^{2}+m^{2}-P_{2}^{2}}{2} \\
\frac{M^{2}+m^{2}-P_{1}^{2}}{2} & \frac{M^{2}+m^{2}-P_{2}^{2}}{2} & m^{2}
\end{array}\right|=0 .
$$

To this manifold in momentum space corresponds a manifold in Feynman parameter space given by the dark shaded area in Fig. 3 .

\section{EFT Dimensional Regularization}

In this section, a natural regularization scheme (EFTDR) for an EFT applicable below a scale $\Lambda_{\mathrm{EFT}}$ is established. It is the low-energy kinematic variables that control the divergences of the loop integrals regularized using EFTDR. The low-energy domain is defined by the relations

$$
\begin{aligned}
\left|P_{v} \cdot P_{v^{\prime}}-M^{2}\right| & \ll \Lambda_{\mathrm{EFT}}^{2}, \\
P_{v} \cdot p_{v^{\prime}}, p_{v} \cdot p_{v^{\prime}}, m^{2} & \ll \Lambda_{\mathrm{EFT}}^{2}, \\
M & \gtrsim \Lambda_{\mathrm{EFT}},
\end{aligned}
$$

where $P_{v}^{\mu}$ and $p_{v}^{\mu}$ are the heavy and light particle external momenta respectively, while $M$ and $m$ are generic heavy and light particle masses respectively.

EFTDR is implemented by consistently separating a loop integral $I_{G}$ corresponding to a graph $G$ into two parts,

$$
I_{G}=\bar{I}_{G}+R_{G},
$$

where the entire low-energy analytic structure of $I_{G}$ is collected into $\bar{I}_{G}$ while all the terms multiplied by factors of the form $M^{\ell d-n}$ (referred to as fractional powers of $M$ for fractional space-time dimension $d$ ) where $\ell$ and $n$ are integers, are collected into $R_{G}$, the regular part. The analytic structure of $\bar{I}_{G}$ and $I_{G}$ are identical in the low-energy domain such that $R_{G}$ can be accounted for through a renormalization of the low-energy constants of the EFT. The separation (18) will be achieved by systematically separating out the subgraphs representing singularities that lie outside of the low-energy region; these subgraphs will be referred to as regular subgraphs.

In the example of the triangle graph, the only singularities that lie outside of the low-energy region are the $M=0$ singularities represented by Figs. 2(e)-(f).

\footnotetext{
${ }^{4}$ The normalization condition (可 cannot be fulfilled.

${ }^{5}$ The terminology is taken from Ref. [6] and refers to the fact that to one-loop, $R_{G}$ is analytic in the low-energy region.
} 


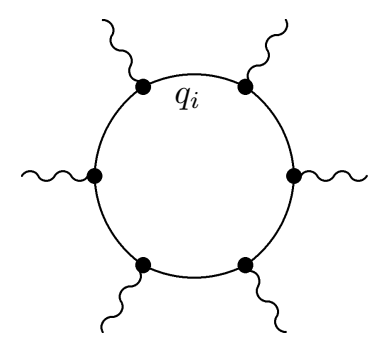

Figure 4: A regular subgraph composed of heavy particle propagators and lowenergy momentum insertions represented by wiggly lines.

Since a factor $M^{\ell d-n}$ will be singular at $M=0$ for appropriate $d$, separating out the $M=0$ singularity will ensure that the terms multiplied by fractional powers of $M$ are separated out.

Generally, subgraphs containing only heavy particle propagators and lowenergy momentum insertions are regular subgraphs that give rise to fractional powers of $M$. Indeed, the Landau equations (7) for the regular subgraph shown in Fig. 囵are

$$
M^{2}\left[1-\sum_{j=1}^{I} \frac{1}{2 M^{2}}\left(q_{i}-q_{j}\right)^{2} \alpha_{j}\right] \approx M^{2}=0 .
$$

Therefore, these subgraphs cannot have singularities in the low-energy region and belong in $R_{G}$.

The subgraphs that lead to fractional powers of $M$ can be isolated by extending the Feynman parameter domain of integration in Eq. (2) where the $\theta\left(\alpha_{k}\right)$ define the integral boundaries. The product of $\theta$ 's can be rewritten:

$$
\begin{aligned}
& \delta\left(1-\sum_{k=1}^{I} \alpha_{k}\right) \prod_{i=1}^{I}\left[1-\theta\left(-\alpha_{i}\right)\right] \\
&=\delta\left(1-\sum_{k=1}^{I} \alpha_{k}\right)\left\{1+\sum_{i=1}^{I}\left[-\theta\left(-\alpha_{i}\right)\right]\right. \\
&\left.+\sum_{\substack{i, j=1 \\
i<j}}^{I}\left[-\theta\left(-\alpha_{i}\right)\right]\left[-\theta\left(-\alpha_{j}\right)\right]+\cdots\right\} .
\end{aligned}
$$

It is noted that each $\theta\left(-\alpha_{i}\right)$ contains the point $\alpha_{i}=0$ which, as discussed in the previous section, represents a contracted line in $G$ that leads to a subgraph 
of $G$. In light of this, each term in Eq. (20) defines a domain of integration $\Delta_{g}$,

$$
\Delta_{g}=\delta\left(1-\sum_{k=1}^{I} \alpha_{k}\right) \prod_{i \notin g}\left[-\theta\left(-\alpha_{i}\right)\right]
$$

which can be associated with a particular subgraph $g$ of $G$ [ $R_{G}$ is then defined as

$$
R_{G}=\mathrm{i}^{I} \Gamma(I) \mu^{L(4-d)} \int_{-\infty}^{\infty} \prod_{k=1}^{I} \mathrm{~d} \alpha_{k} \mathcal{D}_{R_{G}} \int \prod_{\ell=1}^{L} \frac{\mathrm{d}^{d} k_{\ell}}{(2 \pi)^{d}}\left[\sum_{i=1}^{I} \alpha_{i}\left(q_{i}^{2}-m_{i}^{2}\right)+\mathrm{i} \epsilon\right]^{-I},
$$

where

$$
\mathcal{D}_{R_{G}}=\sum_{\text {regular }} \Delta_{g} \text { 's }
$$

It is instructive to discuss the triangle graph $I_{3}$ as an illustration of the method. Tab. 1 shows the association of each term in the $\theta$-expansion with the corresponding subgraph and domain of integration. As mentioned above, the only regular subgraphs are the heavy particle tadpoles located at $\left(\alpha_{1}, \alpha_{2}, \alpha_{3}\right)=$ $(1,0,0)$ and $(0,1,0)$ in Fig 3 . The integration domains in Eq. (20) that contain these points as endpoints are: $\theta\left(-\alpha_{2}\right) \theta\left(-\alpha_{3}\right)$ and $\theta\left(-\alpha_{1}\right) \theta\left(-\alpha_{3}\right)$. These integration domains do not include any of the low-energy singularities; this implies that $R_{3}$ is analytic in the low-energy domain. $\bar{I}_{3}$ is then given by

$$
\bar{I}_{3}=I_{3}-R_{3},
$$

and possesses the same low-energy analytic structure in the small kinematical variables as $I_{3}$ but does not contain any fractional powers of $M$. This is evident by looking at the integration domains corresponding to Eq. (24) and drawn in Fig. 第.
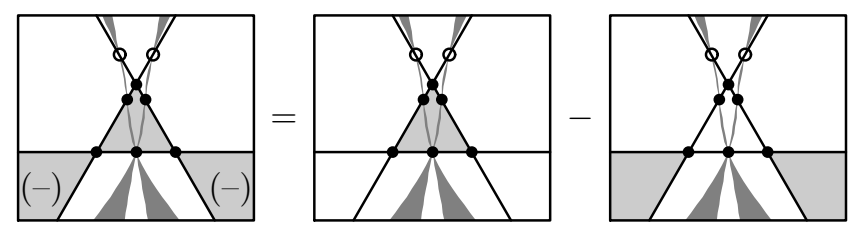

Figure 5: Decomposition of the integration contour for the triangle graph

For a general Feynman graph $G$, a particular domain of integration $\Delta_{g}$ associated with a subgraph $g$ will never select a singularity associated with any subgraph of $g$. Indeed, the domain of integration $\Delta_{g}$ will never have as an

${ }^{6}$ The subscript $g$ represents a set of indices labeling the internal lines of the subgraph $g$ 


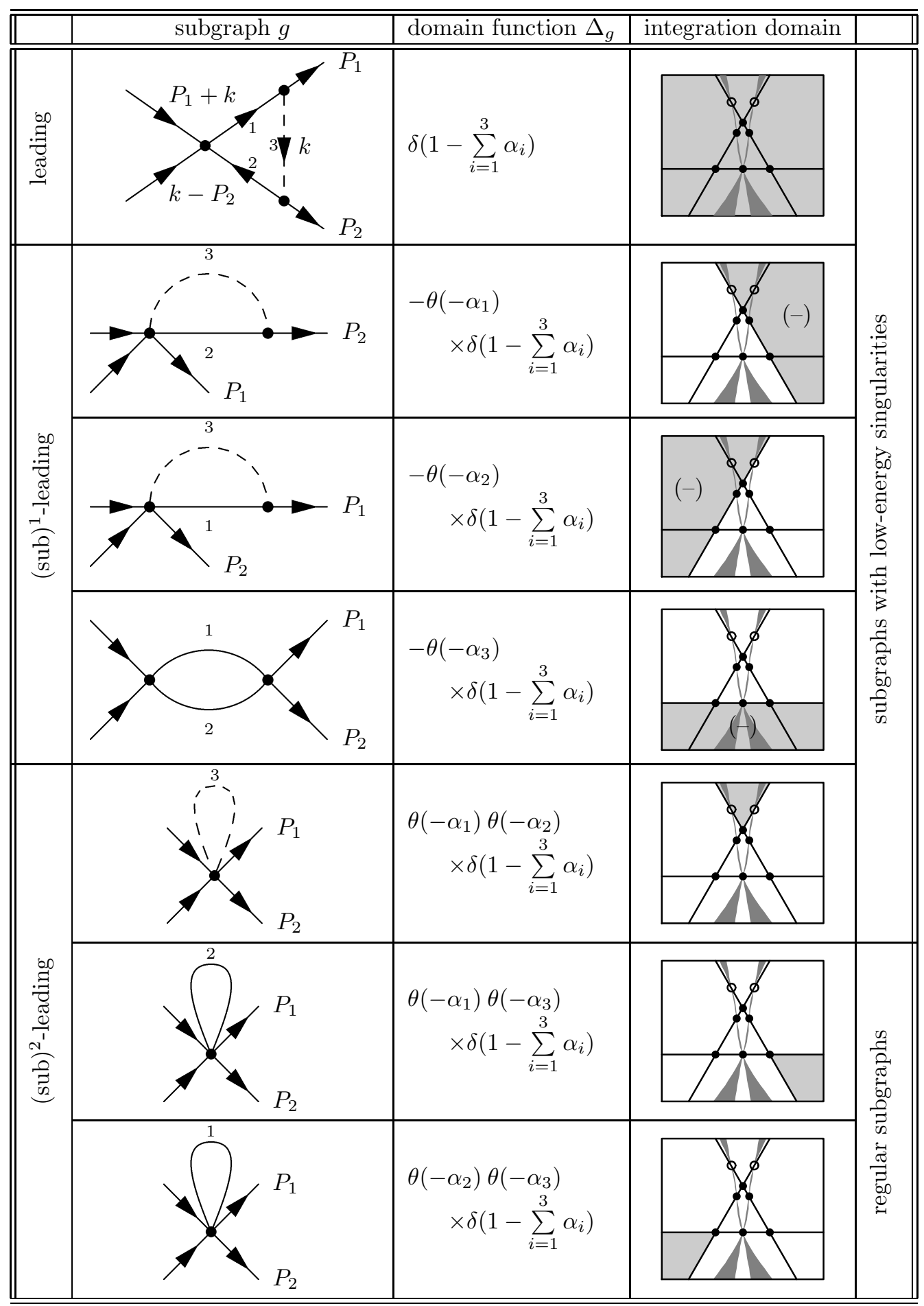

Table 1: Integration domains for the triangle graph 
endpoint the location in $\alpha$-space of any singularity associated with a subgraph of $g$. It follows that a subgraph $\bar{g}$ that contributes to $\bar{I}_{G}$ can never give rise to fractional powers of $M$ since they can only arise from regular subgraphs that are subgraphs of $\bar{g}$. This proves that $R_{G}$ contains all the fractional powers of $M$. To one loop, it is also straightforward to show that $R_{G}$ is analytic in the low-energy region.

\section{$3.1 \quad$ One-loop graphs}

For one-loop graphs, more explicit results can be obtained. Evidently, any subgraph of a regular subgraph, obtained by contracting out further lines, is also regular. This gives rise to the notion of a minimally contracted regular (MCR) subgraph, i.e., a regular subgraph $\mathfrak{g}$ that is not a subgraph of any other regular subgraph with more lines than $\mathfrak{g}$. In EFTs to one-loop order, there are at most two different MCR subgraphs of a given graph and every internal heavy-particle line belongs to exactly one of them (cf. App. A). As an example, the two tadpoles of Figs. 2(e)-(f) are the MCR subgraphs of the triangle graph.

Since by construction different MCR subgraphs do not have common lines, they cannot have subgraphs in common. Any regular subgraph $g$ is thus a subgraph of exactly one MCR subgraph $\mathfrak{g}$. The domain functions (21) of a MCR subgraph $\mathfrak{g}$ and all its subgraphs $g \subset \mathfrak{g}$ can be re-summed to yield

$$
\begin{aligned}
\sum_{g \subseteq \mathfrak{g}} \Delta_{g} & =\delta\left(1-\sum_{k=1}^{I} \alpha_{k}\right)\left\{1-\sum_{i \in \mathfrak{g}} \theta\left(-\alpha_{i}\right)+\cdots\right\} \prod_{j \notin \mathfrak{g}}\left[-\theta\left(-\alpha_{j}\right)\right] \\
& =\delta\left(1-\sum_{k=1}^{I} \alpha_{k}\right) \prod_{i \in \mathfrak{g}} \theta\left(\alpha_{i}\right) \prod_{j \notin \mathfrak{g}}\left[-\theta\left(-\alpha_{j}\right)\right] .
\end{aligned}
$$

The domain function $\mathcal{D}_{R_{G}}$ introduced in Eq. (23) is then

$$
\mathcal{D}_{R_{G}}=\sum_{\mathfrak{g}} \sum_{g \subseteq \mathfrak{g}} \Delta_{g}
$$

and the full regular part is given as

$$
\begin{aligned}
& R_{G}=\mathrm{i}^{I} \Gamma(I) \mu^{4-d} \sum_{\mathfrak{g}} \int_{0}^{\infty} \prod_{i \in \mathfrak{g}} \mathrm{d} \alpha_{i} \int_{-\infty}^{0} \prod_{j \notin \mathfrak{g}}\left(-\mathrm{d} \alpha_{j}\right) \delta\left(1-\sum_{k=1}^{I} \alpha_{k}\right) \\
& \times \int \frac{\mathrm{d}^{d} k}{(2 \pi)^{d}}\left[\sum_{i=1}^{I} \alpha_{i}\left(q_{i}^{2}-m_{i}^{2}\right)+\mathrm{i} \epsilon\right]^{-I} .
\end{aligned}
$$

The momentum integration can be carried out explicitly [10] yielding

$$
R_{G}=\kappa_{I} \sum_{\mathfrak{g}} \int_{0}^{\infty} \prod_{i \in \mathfrak{g}} \mathrm{d} \alpha_{i} \int_{-\infty}^{0} \prod_{j \notin \mathfrak{g}}\left(-\mathrm{d} \alpha_{j}\right) \delta\left(1-\sum_{k=1}^{I} \alpha_{k}\right)[C-\mathrm{i} \epsilon]^{d / 2-I},
$$


where:

$$
C=\sum_{i, j=1}^{I} \alpha_{i} \Omega_{i j} \alpha_{j}, \quad \Omega_{i j}=\frac{m_{i}^{2}+m_{j}^{2}-\left(q_{i}-q_{j}\right)^{2}}{2} .
$$

The matrix $\Omega_{i j}$ was defined in Eq. (8) and the factors have been collected in

$$
\kappa_{I}=(-)^{I} \frac{\mathrm{i}^{I+1}}{16 \pi^{2}} \frac{\Gamma(I-d / 2)}{\left(4 \pi \mu^{2}\right)^{d / 2-2}} .
$$

A useful representation of $R_{G}$ with a single non-compact integral can be derived by inserting the identity

$$
1=\int_{1}^{\infty} \mathrm{d} \lambda \delta\left(\lambda-\sum_{k \in \mathfrak{g}} \alpha_{k}\right)
$$

into Eq. (29) and performing the change of variables

$$
\begin{aligned}
\alpha_{i} & =\lambda z_{i} & & i \in \mathfrak{g}, \\
\alpha_{i} & =(1-\lambda) z_{i} & & i \notin \mathfrak{g} .
\end{aligned}
$$

This yields for $R_{G}$ :

$$
\begin{aligned}
R_{G}=-\kappa_{I} \sum_{\mathfrak{g}} \int_{1}^{\infty} \mathrm{d} \lambda \lambda^{|\mathfrak{g}|-1}(1-\lambda)^{I-|\mathfrak{g}|-1} & \times \int_{0}^{1}\left(\prod_{i \in \mathfrak{g}} \mathrm{d} z_{i}\right) \delta\left(1-\sum_{k \in \mathfrak{g}} z_{k}\right) \int_{0}^{1}\left(\prod_{j \notin \mathfrak{g}} \mathrm{d} z_{j}\right) \delta\left(1-\sum_{k \notin \mathfrak{g}} z_{k}\right) \\
& \times\left[(1-\lambda)^{2} \sum_{i, j \notin \mathfrak{g}} z_{i} \Omega_{i j} z_{j}+\lambda^{2} \sum_{i, j \in \mathfrak{g}} z_{i} \Omega_{i j} z_{j}\right. \\
& \left.+2 \lambda(1-\lambda) \sum_{\substack{i \in \mathfrak{g} \\
j \notin \mathfrak{g}}} z_{i} \Omega_{i j} z_{j}-\mathrm{i} \epsilon\right]^{d / 2-I},
\end{aligned}
$$

where $|\mathfrak{g}|$ denotes the number of internal lines belonging to the MCR subgraph $\mathfrak{g}$.

In this representation, it is straightforward to prove that $R_{G}$ is analytic in the low-energy region. As stated above, every internal heavy-particle line belongs to one of the two MCR subgraphs $\mathfrak{g}_{1}$ or $\mathfrak{g}_{2}$.] Denoting the set of internal lightparticle lines by $\mathfrak{l}$, the elements of the matrix appearing in Eq. (30) are (cf. Eq. (41) in App. A

$$
\Omega_{i j}=\left\{\begin{array}{ll}
\mathcal{O}\left(p^{2}\right) & i, j \in \mathfrak{l} \\
\mathcal{O}(p) M & i \in \mathfrak{l}, \quad j \notin \mathfrak{l} \\
M^{2}+\mathcal{O}\left(p^{2}\right) & i, j \in \mathfrak{g}_{1} \quad \text { or } \quad i, j \in \mathfrak{g}_{2} \\
-M^{2}+\mathcal{O}(p) M & i \in \mathfrak{g}_{1}, \quad j \in \mathfrak{g}_{2}
\end{array},\right.
$$

\footnotetext{
${ }^{7}$ Here $\mathfrak{g}_{2}$ is understood to be empty in cases where there is only one MCR subgraph.
} 
yielding for the denominator of Eq. (34) with $\mathfrak{g}=\mathfrak{g}_{1}$ :

$$
\begin{gathered}
\sum_{i, j=1}^{I} \alpha_{i} \Omega_{i j} \alpha_{j}=(1-\lambda)^{2} \sum_{i, j \in \mathfrak{g}_{2}} z_{i} M^{2} z_{j}-2 \lambda(1-\lambda) \sum_{\substack{i \in \mathfrak{g}_{1} \\
j \in \mathfrak{g}_{2}}} z_{i} M^{2} z_{j} \\
+\lambda^{2} \sum_{i, j \in \mathfrak{g}_{1}} z_{i} M^{2} z_{j}+\mathcal{O}(p) M \\
=M^{2}(1+\nu)^{2} \lambda^{2}\left[1-\frac{\nu}{(1+\nu) \lambda}\right]^{2}+\mathcal{O}(p) M,
\end{gathered}
$$

where $\nu=\sum_{i \in \mathfrak{g}_{2}} z_{i}$ with $0 \leq \nu \leq 1$ and $\lambda \geq 1$. Thus, the denominator never vanishes proving that $R_{G}$ is always analytic for one-loop graphs with arbitrary numbers of heavy and light particle propagators. The integral over $\lambda$ can be performed by expanding the square bracket in Eq. (36). To leading order in this expansion, this gives

$$
\begin{aligned}
& R_{G} \simeq \sum_{\mathfrak{g}} \frac{(-1)^{I-|\mathfrak{g}|-1} M^{d-2 I} \kappa_{I}}{(d-}I-1) \Gamma(|\mathfrak{g}|) \\
& \quad \times \int_{0}^{1}\left(\prod_{i \notin \mathfrak{g}} \mathrm{d} z_{i}\right) \delta\left(1-\sum_{k \notin \mathfrak{g}} z_{k}\right)(1+\nu)^{d-2 I}+M \mathcal{O}(p),
\end{aligned}
$$

where the $\lambda$-integral was performed at $d$ sufficiently small to drop the upper boundary; $d$ can now be analytically continued to 4 space-time dimensions. Eq. (37) can be used to calculate to leading order the regular part of $R_{3}$ keeping in mind that there are two MCR subgraphs for the triangle graph:

$$
R_{3}=-\frac{1}{16 \pi^{2}} \frac{\Gamma(3-d / 2)}{\left(4 \pi \mu^{2}\right)^{d / 2-2}} \frac{M^{d-6}}{(d-4)(d-5)}(1+\cdots) .
$$

Eq. (38) agrees with the result given in Ref. [8].

Eq. (37) explicitly shows that $R_{G}$ is proportional to a fractional power of $M$. This implies that $R_{G}$ and $\bar{I}_{G}$ transform separately under the symmetry transformations that leave the Lagrangian invariant [6]. This ensures that EFTDR preserves all the symmetries of the underlying Lagrangian. For general one-loop graphs, Eq. (37) also shows that the non-compact integrals appearing in $\bar{I}_{G}$ can be performed quite generally.

The Taylor series expansion of $R_{G}$ in powers of $p$ is truncated at the order of the EFT Lagrangian. It follows that the Taylor-polynomial of $R_{G}$ can be absorbed into the low-energy constants of the EFT Lagrangian. Once $R_{G}$ has been used to define the domain of integration of $\bar{I}_{G}$, it need not be considered further.

For a general one-loop Feynman graph $G$, the EFT dimensional regularization scheme is summarized by the following simple rules: 
1. Label all of the internal lines $i$ of the graph $G$ by the corresponding Feynman parameter $\alpha_{i}$.

2. Determine the minimally contracted regular subgraph(s) $\mathfrak{g}$ of $G$.

3. For each minimally contracted regular subgraph $\mathfrak{g}$, integrate the Feynman parameters corresponding to internal lines of $\mathfrak{g}$ from 0 to $+\infty$; integrate the remaining Feynman parameters from $-\infty$ to 0 inserting a factor of -1 for every integral.

4. Sum these contributions for all minimally contracted subgraphs to obtain the regular part $R_{G}$.

5. The EFT dimensionally regularized Feynman integral is given as

$$
\bar{I}_{G}=I_{G}-R_{G} .
$$

\subsection{Discussion of multi-loop graphs}

It is emphasized that the regularization scheme developed above is not limited to one-loop graphs. Indeed, the Landau equations are valid to arbitrary number of loops and allow a determination of those subgraphs that give rise to fractional powers of $M$. The contributions from these and only these subgraphs are collected in $R_{G}$ and need to be separated from the dimensionally regularized Feynman integral $I_{G}$ to obtain the EFT dimensionally regularized integral $\bar{I}_{G}$. The separation

$$
I_{G}=\bar{I}_{G}+R_{G}
$$

is again achieved by extending the domain of integration as explained above. As a novel feature at two or more loops, subgraphs that give rise to fractional powers of $M$ may now contain light internal lines and may give rise to lowenergy singularities. An example of such a subgraph is a tadpole attached to a triangle graph as shown in Fig. 6(a): the tadpole part of the graph gives rise to fractional powers of $M$, while the triangle part contains low-energy singularities. The appearance of these graphs does not constitute a problem since their lowenergy singularities are not new types of singularities but rather originate from a lower number of loops. Even after separating out $R_{G}$, all the thresholds and the associated analytic structure of these low-energy singularities are still contained in the low-energy part $\bar{I}_{G}$ through subgraphs with fewer loops. In the two-loop example shown in Fig. 6, the low-energy singularities are identical to those of the one-loop triangle graph which are retained in $\bar{I}_{G}$ according to the regularization scheme and the contribution of Fig. 6 corresponds to the triangle graph with a renormalized four-heavy-particle vertex shown in Fig. 6(b). 


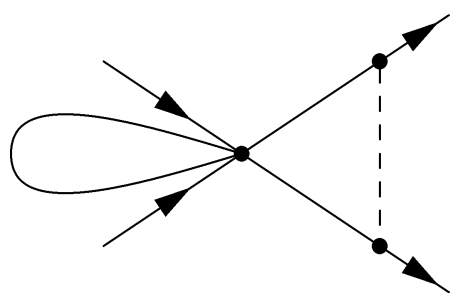

(a)

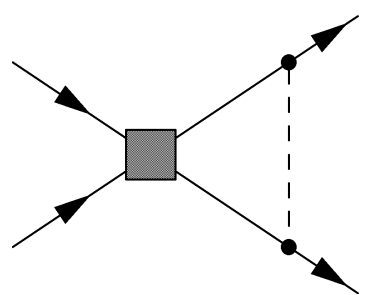

(b)

Figure 6: Figure (a) shows an example of an unnested two-loop graph that gives rise to fractional powers of $M$ while containing low-energy singularities. Figure (b) shows the triangle graph with renormalized four-heavy-particle vertex.

\section{Summary}

In summary, a manifestly Lorentz-invariant regularization scheme which does not violate power counting was derived. This scheme separates a Feynman integral $I_{G}$ represented by a Feynman graph $G$ into two parts, $I_{G}=\bar{I}_{G}+R_{G}$, and is referred to as EFTDR; the entire low-energy analytic structure of $I_{G}$ is collected into $\bar{I}_{G}$ while all the low-energy power counting violating contributions are collected into the regular part $R_{G}$. This separation is achieved by identifying the power counting violating contributions to particular solutions of the Landau equations which are represented by subgraphs of $G$. In EFTDR, each subgraph $g$ of $G$ is associated with a domain of integration $\Delta_{g}$ in the space of Feynman parameters such that the power counting violating terms are separated out by extending the domain of integration. Simple diagrammatic rules were given which allow the construction of $\bar{I}_{G}$ (and $R_{G}$ ) for one-loop diagrams with an arbitrary number of heavy propagating particles. $R_{G}$ can be accounted through a renormalization of the LECs of the EFT Lagrangian and discarded; the EFTDR regularized Feynman graph $I_{G}$ is then $\bar{I}_{G}$. It was shown that $R_{G}$ and $\bar{I}_{G}$ transform separately under the transformations that leave the EFT Lagrangian invariant such that all the Ward identities are satisfied by the EFTDR regularized Greens functions of the EFT. The application of EFTDR to higher loops was discussed and it was emphasized that the power counting violating contributions can still be separated out systematically by extending the domain of integration in the space of Feynman parameters. EFTDR was applied to the one-heavy particle and two-heavy particle sectors to one loop and shown to reproduce the results in Refs. [6, 8].

\section{Acknowledgments}

The authors would like to thank J. L. Goity for his support and J. D. Walecka for valuable comments on the manuscript. They would also like to thank Thomas 


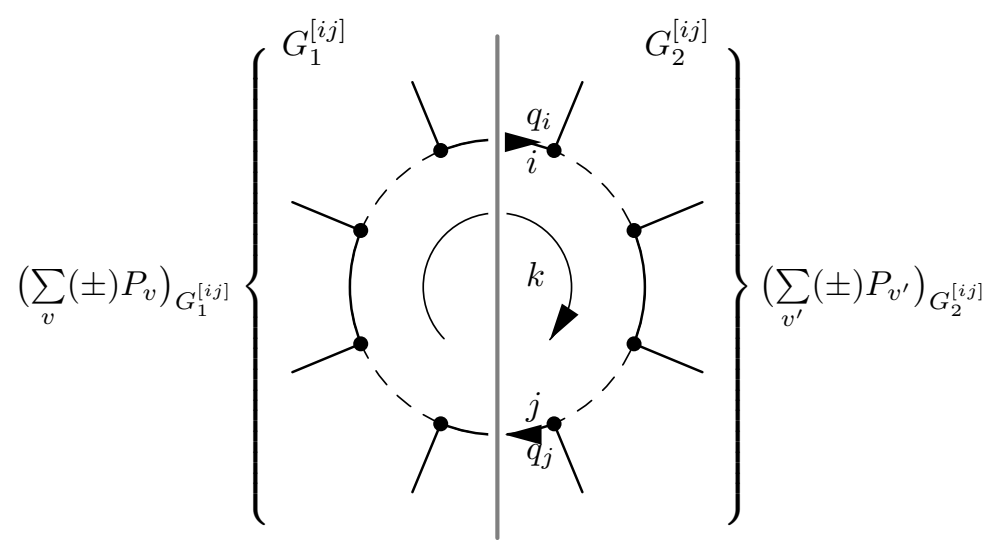

Figure 7: Cutting a one-loop graph

Jefferson National Accelerator Facility (Jefferson Lab) for their hospitality. This work was supported by the National Science Foundation through grant \# HRD9633750 (DL and GP) and by the Deutsche Forschungsgemeinschaft through project Le 1189/1-1 (DL).

\section{A Minimally contracted regular subgraphs}

Consider a general one-loop graph $G$ formed with heavy (mass $M$ ) and light (mass $m$ ) particles and external momenta $P_{v}^{\mu}$. By cutting two different lines $i$ and $j$ the graph is divided into two connected parts $G_{1}^{[i j]}$ and $G_{2}^{[i j]}$, see Fig. 7. Four-momentum conservation at each vertex of $G$ implies that the squared momentum flowing through the cut, $\left(q_{i}-q_{j}\right)^{2}$, equals the squared total external momentum attached to either one of the two pieces,

$$
\left(q_{i}-q_{j}\right)^{2}=\left(\sum_{v}( \pm) P_{v}\right)_{G_{1}^{[i j]}}^{2}=\left(-\sum_{v^{\prime}}( \pm) P_{v^{\prime}}\right)_{G_{2}^{[i j]}}^{2}
$$

where the momenta $q_{i}^{\mu}$ of internal lines are routed to flow around the loop and the sign of the external momenta $P_{v}^{\mu}$ depends on whether the momenta are incoming or outgoing. Since in an EFT all external three-momenta are $\mathcal{O}\left(p^{2}\right) \ll \Lambda_{\mathrm{EFT}}$ and creation/annihilation of heavy particles is excluded, it fol- 


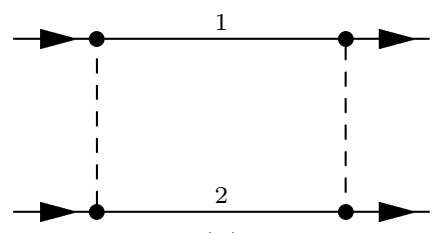

(a)

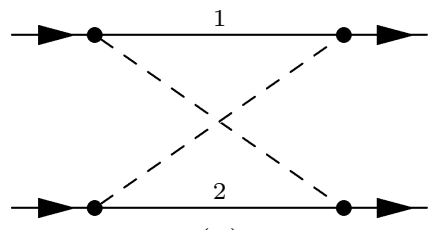

(b)

Figure 8: Box graph (a) and cross box graph (b)

lows immediately that

$$
\left(q_{i}-q_{j}\right)^{2}=\left\{\begin{array}{lll}
\mathcal{O}\left(p^{2}\right) & i, j \text { light } \\
M^{2}+\mathcal{O}(p) M & i \text { light, } j \text { heavy } \\
(2 M)^{2}+\mathcal{O}(p) M & i, j \text { heavy } & {[\mathrm{a}]} \\
\mathcal{O}\left(p^{2}\right) & i, j \text { heavy } & {[\mathrm{b}]}
\end{array}\right.
$$

An example for the case (41a) is found when cutting the two heavy-particle lines of the box graph Fig. $8(a)$, while the crossed box graph Fig. 8 (b) corresponds to case (41b).

The crucial observation is now that for any three heavy-particle internal lines $i$, $j$, and $k$ :

$$
\left.\begin{array}{l}
\left(q_{i}-q_{j}\right)^{2}=\mathcal{O}\left(p^{2}\right) \\
\text { and } \\
\left(q_{j}-q_{k}\right)^{2}=\mathcal{O}\left(p^{2}\right)
\end{array}\right\} \Rightarrow\left(q_{i}-q_{k}\right)^{2}=\mathcal{O}\left(p^{2}\right)
$$

This transitivity relation follows from Eq. (40) and

$$
\left(-\sum_{v}( \pm) P_{v}\right)_{G_{2}^{[i k]}}=\left(\sum_{v}( \pm) P_{v}\right)_{G_{2}^{[i j]}}+\left(\sum_{v}( \pm) P_{v}\right)_{G_{2}^{[j k]}}
$$

see Fig. 9. Any two heavy-particle lines $i$ and $j$ of a graph $G$ are said to belong to the same minimally contracted regular (MCR) subgraph $\mathfrak{g}$ if and only if they satisfy condition Eq. (41b),

$$
i, j \in \mathfrak{g} \quad: \Leftrightarrow\left(q_{i}-q_{j}\right)^{2}=\mathcal{O}\left(p^{2}\right) .
$$

This defines the MCR subgraphs of $G$ completely. In light of Eq. (42), different MCR subgraphs cannot have any internal lines in common. On the other hand, every heavy-particle internal line belongs at least to one regular subgraph, namely the tadpole graph corresponding to that line. Therefore, the set of heavy-particle internal lines of a graph $G$ can be split into disjoint subsets; each subset is diagrammatically represented by a subgraph $\mathfrak{g}$ of $G$ that consists

\footnotetext{
${ }^{8}$ It is to be noted that Eq. (41) remains true for all subgraphs of $G$. Indeed, the l.h.s. is unaffected by the contraction lines to a point.
} 


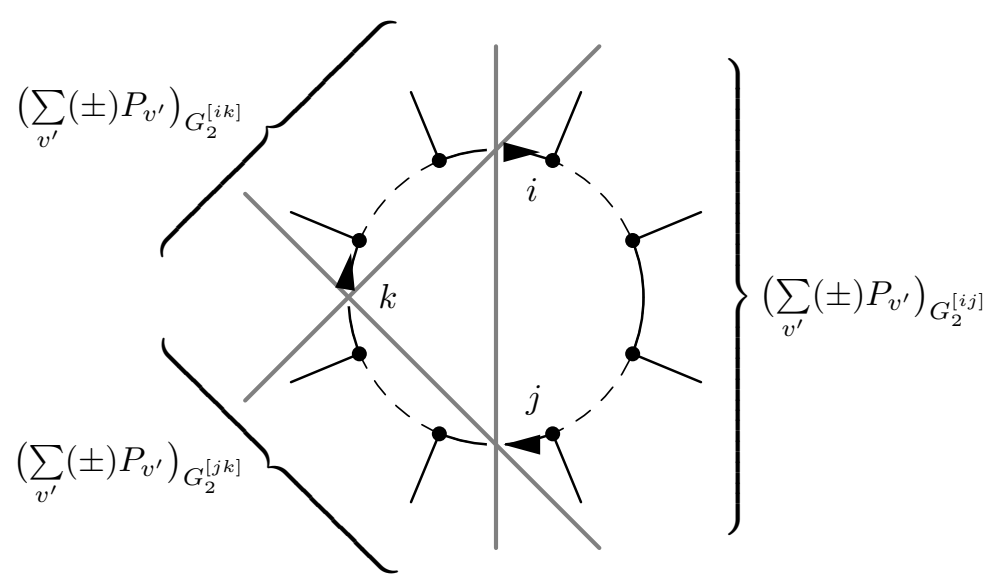

Figure 9: Transitivity of cuts

of all the lines belonging to the subset and of no other lines. These subgraphs are the MCR subgraphs. They do not have low-energy singularities, since by construction all (effective) external momenta are small while all internal lines are heavy.

In the box graph Fig. \&(a) the two heavy-particle lines 1 and 2 do not belong to the same MCR subgraph; in this case the tadpole graphs formed with lines 1 and 2 are both MCR subgraphs. In the cross box graph Fig. 8(b), however, there is only one MCR subgraph, namely the bubble graph formed with lines 1 and 2 .

It is noted that at most two different MCR subgraphs of a given one-loop graph exist. This can be seen as follows: assume the existence of more than two different MCR subgraphs and choose three heavy internal lines $i, j$, and $k$ belonging to different MCR subgraphs. It is easily verified that

$$
\left.\begin{array}{l}
\left(q_{i}-q_{j}\right)^{2}=4 M^{2}+\mathcal{O}(p) M \\
\text { and } \\
\left(q_{j}-q_{k}\right)^{2}=4 M^{2}+\mathcal{O}(p) M
\end{array}\right\} \Rightarrow\left(q_{i}-q_{k}\right)^{2} \neq 4 M^{2}+\mathcal{O}(p) M
$$

However, since $\left(q_{i}-q_{k}\right)^{2}$ must satisfy Eq. (41), lines $i$ and $k$ have to belong to the same MCR subgraph,

$$
\left(q_{i}-q_{k}\right)^{2}=\mathcal{O}\left(p^{2}\right),
$$

in contradiction to the above assumption.

\section{B One-loop examples}

This formalism reproduces the prescriptions given for the one-loop graphs in Figs. 10(a) and 11.(a) first derived in Refs. [6, 8]. Fig. 10(a) is the most general 


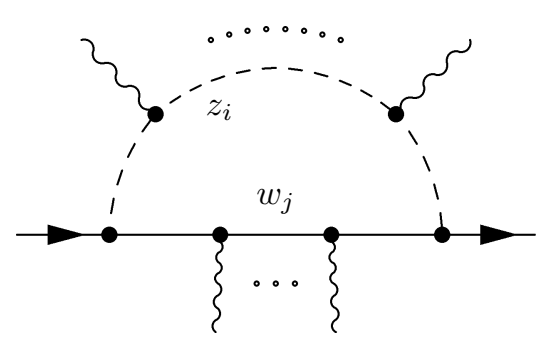

(a)

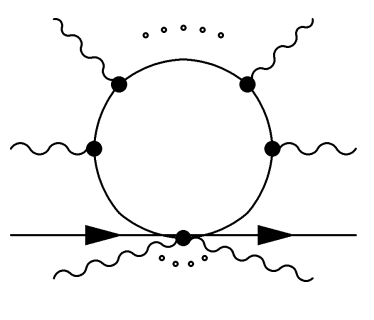

(b)

Figure 10: (a) General one-loop graph with one heavy particle. (b) The corresponding minimally contracted regular subgraph.

one-loop graph with a single heavy particle line running through it; there are $H-1$ soft momentum insertions entering the heavy particle internal line and $l-1$ soft momentum insertions entering the light particle internal line.

The regular part for the graph in Fig. 10(a) corresponds to the MCR subgraph in Fig. 10(b) where all the light internal lines have been contracted. Eq. (34) gives

$$
\begin{aligned}
R & =-\kappa_{I} \int_{1}^{\infty} \mathrm{d} \lambda \lambda^{H-1}(1-\lambda)^{l-1} \\
& \times \int_{0}^{1}\left(\prod_{i=1}^{l} \mathrm{~d} z_{i}\right)\left(\prod_{j=1}^{H} \mathrm{~d} w_{j}\right) \delta\left(1-\sum_{k=1}^{l} z_{k}\right) \delta\left(1-\sum_{k=1}^{H} w_{k}\right)[C-\mathrm{i} \epsilon]^{d / 2-I},
\end{aligned}
$$

which is the result first obtained by Becher and Leutwyler [6] for a one-loop graph with $l$ light particle propagators labeled $1, \ldots, l$ and $H$ heavy particle propagators labeled $l+1, \ldots, I$ where $I=l+H$. Indeed, Eq. (47) describes a Feynman parameterization where all the light particle propagators were combined into a single denominator, all the heavy particle propagators were combined into another denominator, and these two denominators were subsequently combined with the Feynman parameter $\lambda$ integrated between one and infinity; this is precisely the regular part in Becher-Leutwyler's infrared regularization.

For a Feynman graph with two heavy particle lines running through it, Figs. 11.(a)-(b) are the most general diagrams that can be drawn. I] For the Feynman graph shown in Fig. 11(a), there are two MCR subgraphs obtained by contracting all the light particle internal lines as well as one of the heavy internal line running through the graph. The regular part is given by the sum of the MCR subgraphs. Assuming $l$ light particle internal lines and that the top heavy particle internal line is segmented by $h_{1}-1$ soft momentum insertions

\footnotetext{
${ }^{9}$ For example, the triangle graph is obtained from Fig. 11. (a) by removing one of the light particle internal lines that runs through the graph.
} 


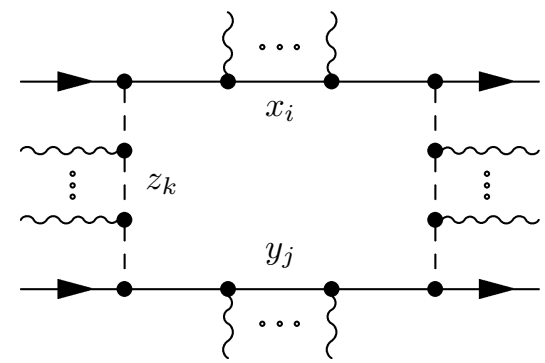

(a)

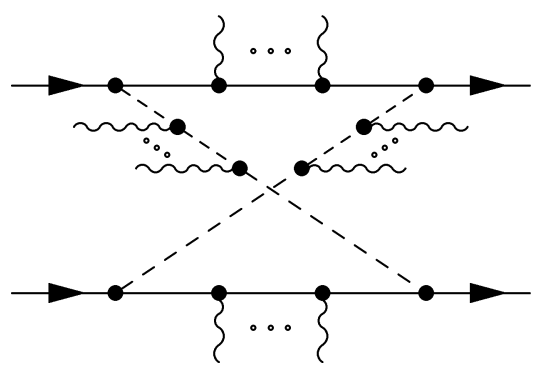

(b)

Figure 11: (a) Generalized box graph. (b) Generalized cross box graph.

while the bottom heavy particle internal line is segmented by $h_{2}-1$ soft momentum insertions, a change of variables similar to Eq. (33) can be performed on Eq. (34) to cast it in the form in which it appears in Ref. [8]. For the MCR obtained by contracting the $h_{n}(n=1,2)$ propagators of one of the heavy lines, inserting the integrals

$$
1=\int_{1}^{\infty} \mathrm{d} \lambda \mathrm{d} \beta \delta\left(\lambda-\sum_{k=l+1}^{I} \alpha_{k}\right) \delta\left(\beta-\frac{1}{\lambda} \sum_{k=l+h_{n}+1}^{I} \alpha_{k}\right)
$$

and performing the change of variables

$$
\begin{array}{rlrl}
\alpha_{i} & =(1-\lambda) z_{i} & & i=1, \ldots, l \\
\alpha_{i+l} & =\lambda(1-\beta) x_{i} & & i=1, \ldots, h_{n} \\
\alpha_{i+l+h_{n}} & =\lambda \beta y_{i} & i & =1, \ldots, H-h_{n}
\end{array}
$$

gives

$$
\begin{aligned}
& R=\sum_{n=1,2}-\kappa_{I} \int_{1}^{\infty} \mathrm{d} \lambda \int_{1}^{\infty} \mathrm{d} \beta \lambda^{H-1}(1-\lambda)^{l-1} \beta^{H-h_{n}-1}(1-\beta)^{h_{n}-1} \\
& \times \int_{0}^{1}\left(\prod_{i=1}^{h_{n}} \mathrm{~d} x_{i}\right)\left(\prod_{j=1}^{H-h_{n}} \mathrm{~d} y_{j}\right)\left(\prod_{k=1}^{l} \mathrm{~d} z_{k}\right) \\
& \times \delta\left(1-\sum_{a=1}^{l} z_{a}\right) \delta\left(1-\sum_{b=1}^{H-h_{n}} y_{b}\right) \delta\left(1-\sum_{c=1}^{h_{n}} x_{c}\right)[C-\mathrm{i} \epsilon]^{d / 2-I},
\end{aligned}
$$

where $H=h_{1}+h_{2}$. This is just the regular part given in [8]. The generalized cross box graph in Fig. 11(b) does not have a two-heavy particle threshold, and its regular part is constructed from a single MCR subgraph obtained by contracting all the light internal lines. 


\section{References}

[1] S. Weinberg, The Quantum Theory of Fields II, (Cambridge University Press, Cambridge, 1996).

J. F. Donoghue, E. Golowich, and B. R. Holstein, Dynamics of the Standard Model (Cambridge University Press, Cambridge, 1996).

[2] S. Weinberg, Physica A96, 327 (1979).

[3] J. Gasser, M. Sainio, and A. Švarc, Nucl. Phys. B307 (1988) 779.

[4] E. Eichten and B. Hill, Phys. Lett. B234 (1990) 511.

H. Georgi, Phys. Lett. B240 (1990) 447.

B. Grinstein, Nucl. Phys. B339 (1990) 253.

E. Jenkins and A. M. Manohar, Phys. Lett. B255 (1991) 558.

[5] V. Bernard, N. Kaiser, and U.-G. Meißner, Int. J. Mod. Phys. E4 (1995) 193.

[6] T. Becher and H. Leutwyler, Eur. Phys. J. C9 (1999) 643, hep-ph/9901384.

[7] P. J. Ellis and H.-B. Tang, Phys. Rev. C57 (1998).

[8] J. L. Goity, D. Lehmann, G. Prézeau, and J. Saez, hep-ph/0101011, submitted to Phys. Lett.

[9] R. J. Eden, P. V. Landshoff, D. I. Olive, and J. C. Polkinghorne, The analytic S-matrix (Cambridge University Press, Cambridge, 1966).

[10] C. Itzykson and J.-B. Zuber, Quantum Field Theory (McGraw-Hill, New York, 1980). 